\title{
LETTER
}

\section{Ultrafast spin dynamics in ferrimagnets with compensation point}

To cite this article: M D Davydova et al 2019 J. Phys.: Condens. Matter 32 01LT01

View the article online for updates and enhancements.

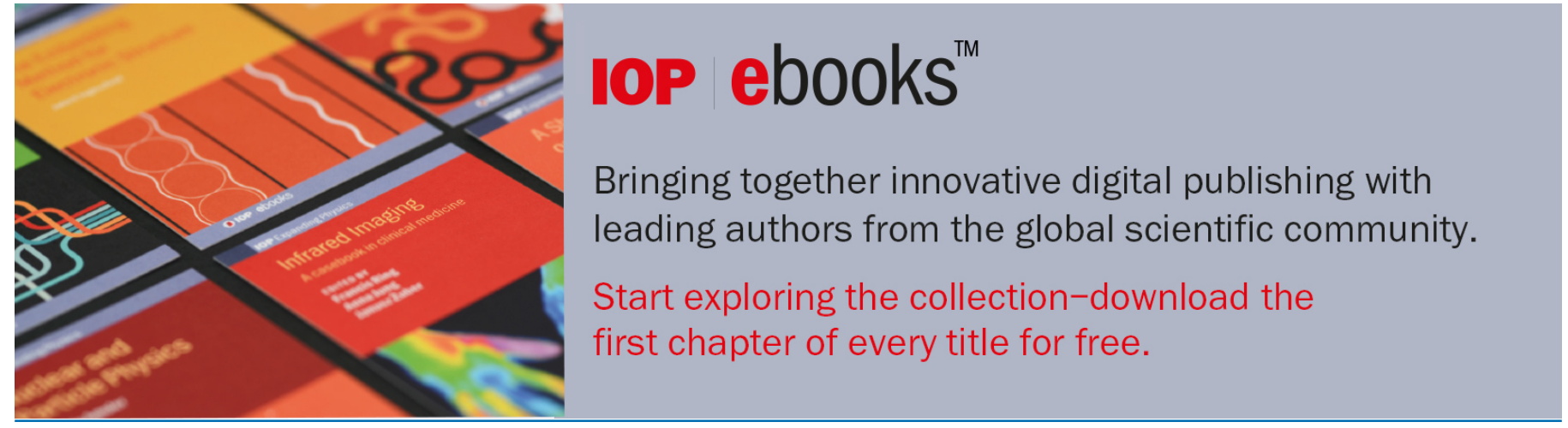

This content was downloaded from IP address 195.169 .220 .16 on $22 / 10 / 2020$ at $14: 33$ 


\title{
Letter
}

\section{Ultrafast spin dynamics in ferrimagnets with compensation point}

\author{
M D Davydova ${ }^{1}$, K A Zvezdin ${ }^{1}$, A V Kimel ${ }^{2,3}$ and A K Zvezdin ${ }^{1,4,5}$ \\ 1 Prokhorov General Physics Institute of the Russian Academy of Sciences, 119991 Moscow, Russia \\ 2 Russian Technological University (MIREA), 119454 Moscow, Russia \\ ${ }^{3}$ Institute for Molecules and Materials, Radboud University, Nijmegen 6525 AJ, The Netherlands \\ ${ }^{4}$ HSE University, 101000 Moscow, Russia \\ E-mail: zvezdin@gmail.com
}

Received 13 May 2019, revised 22 August 2019

Accepted for publication 10 September 2019

Published 11 October 2019

\begin{abstract}
We derive an effective Lagrangian that facilitates the modeling of magnetization dynamics in a ferrimagnet with magnetization compensation point. The model is able to explain the earlier reported magnetization dynamics in the noncollinear magnetic phase triggered by a femtosecond laser pulse in GdFeCo amorphous alloy in the vicinity of spin-flop transition. Moreover, the described approach can be easily extended and applied to other cases of ultrafast magnetism in uniaxial $f$ - $d$ (rare-earth-transition metal) ferrimagnet near the magnetization compensation point in high magnetic fields.

We assume that the primary effect of the femtosecond laser pulse is the ultrafast demagnetization of the ferrimagnet. We show that in the noncollinear magnetic phase, which can be prepared by applying external magnetic field above the spin-flop transition, such a demagnetization results in a torque acting on the magnetizations of both sublattices. It is shown that, similarly to the experiment, the amplitude and timescales of the dynamics strongly depend on temperature and applied magnetic field. In particular, in the vicinity of the spin-flop phase transition the amplitude dramatically increases while the dynamics exhibit a critical slowdown.

We expect that the developed theoretical framework will boost further research of ultrafast magnetism of noncollinear spin systems.
\end{abstract}

Keywords: ultrafast magnetism, rare-earth ferrimagnets, magnetization compensation point, critical response, spin-flop phase transition

S Supplementary material for this article is available online

(Some figures may appear in colour only in the online journal)

\section{Introduction}

Fundamental interest in ever faster magnetization dynamics motivated experiments that use femtosecond laser pulses- the shortest stimulus available in condensed matter physics-and launched the field of ultrafast magnetism [1]. The discovery of ultrafast laser-induced demanetization of ferromagnetic Ni by

\footnotetext{
${ }^{5}$ Author to whom all correspondence should be addressed.
}

Beaurepaire et al [2] and ultrafast all-optical magnetic switching of ferrimagnetic GdFeCo by Stanciu et al [3] challenged the theories in magnetism of that time. The recent developments of atomistic and macrospin simulations allowed to substantially advance in understanding of ultrafast magnetism [4-8]. Application of the existing tools to model multisublattice ferrimagnets such as $\mathrm{GdFeCo}$ revealed new routes for strongly nonequilibrium spin dynamics $[6,9,10]$ and led to the discovery of a counter-intuitive mechanism of magnetization 
reversal by ultrafast heating $[5,11]$. Despite this progress, modeling of ultrafast magnetism in ferrimagnets has usually been limited to the case of collinear magnetic phase.

In most of rare-earth-transition metal (RE-TM) alloys and intermetallics the exchange coupling between the RE and the TM magnetic sublattices is antiferromagnetic and favors a collinear antiparallel alignment in weak magnetic fields. A noncollinear magnetic state in a ferrimagnet can be prepared by applying an external magnetic field that is higher than a certain value. This field is sometimes referred to as spin-flop transition field $H_{\text {sf }}$, analogously to the situation in antiferromagnets [12]. At spin-flop field, ferrimagnet undergoes a phase transition, and the magnetizations of the sublattices turn from the easy magnetization axis, get canted, and form a noncollinear state. In RE-TM ferrimagnets, one finds that it might be possible to find a temperature $T_{M}$, called magnetization compensation temperature, at which the magnetizations of the two antiferromagnetically coupled sublattices mutually compensate one another and the total magnetization of the material turns to zero. The spin-flop field takes the lowest values in the vicinity of this temperature [13]. First experimental reports on ultrafast magnetism in the noncollinear phase revealed very special features [14-16] and rather anomalous behavior [17]. A dramatic increase in amplitude was revealed in combination with a critical slowdown of the dynamics near certain values of magnetic field. These anomalies have not been explained theoretically yet.

In this work, we propose a theoretical framework for calculating magnetization dynamics in a uniaxial $f$-d (RE-TM) ferrimagnet near the compensation point in high magnetic field. The approach is suitable for modeling fast response of magnetization dynamics triggered by femtosecond or picosecond laser pulses. In the model we assume that the primary effect of the pulse is ultrafast demagnetization. The same effect can also be achieved by picosecond electrical current pulses [18, 19] We present a theoretical model and calculations, which allow to describe the ultrafast coherent response of the system that resides in a noncollinear phase before the impact. We show that in this phase the launched magnetization dynamics may become greatly nonlinear being governed by large intersublattice exchange field [20]. We derive the effective Lagrangian that is able to describe the dynamics of the system near the compensation point and obtain the torque acting on the magnetizations of the two sublattices due to the demagnetization. As a particular example in our calculations we use $\mathrm{GdFeCo}$ ferrimagnet. We show that the proposed model explains experimentally observed anomalies and thus represents an efficient tool to explore the area of ultrafast magnetization dynamics in noncollinear ferrimagnets.

\section{Effective Lagrangian and Rayleigh disspation function}

Our approach is based on Landau-Lifshitz-Gilbert equations for a two-sublattice RE-TM ferrimagnet. These equations are equivalent to the following effective Lagrangian and Rayleigh dissipation functions:

$$
\begin{gathered}
\mathcal{L}=-\frac{M_{f}}{\gamma} \cos \theta_{f} \frac{\partial \varphi_{f}}{\partial t}-\frac{M_{d}}{\gamma} \cos \theta_{d} \frac{\partial \varphi_{d}}{\partial t}-\Phi\left(\boldsymbol{M}_{f}, \boldsymbol{M}_{d}, \boldsymbol{H}\right) \\
\mathcal{R}=\mathcal{R}_{f}+\mathcal{R}_{d}, \quad \mathcal{R}_{f, d}=\frac{\alpha M_{f, d}}{2 \gamma}\left(\dot{\theta}_{f, d}^{2}+\sin ^{2} \theta_{f, d} \dot{\varphi}_{f, d}^{2}\right) .
\end{gathered}
$$

Please note that magnetic RE ions are in $f$-state, whereas $\mathrm{Fe}$ and $\mathrm{Co}$ are $d$-ions, and the magnetic subsystems are regarded to as $f$ - and $d$-sublattice, respectively. In equations (1) and (2) $\gamma$ is the gyromagnetic ratio, $\alpha$ is the Gibert damping constant, $\boldsymbol{M}_{d}$ and $\boldsymbol{M}_{f}$ are the magnetizations, $\theta_{d}(\mathrm{TM})$ and $\theta_{f}(\mathrm{RE})$ are the polar, $\varphi_{d}$ and $\varphi_{f}$ are the azimuthal angles of $d$ - and $f$-sublattices correspondingly in the spherical system of coordinates with the $z$-axis aligned with the external magnetic field $\boldsymbol{H}$. $\Phi\left(\boldsymbol{M}_{f}, \boldsymbol{M}_{d}, \boldsymbol{H}\right)$ is the thermodynamic potential for the system:

$$
\Phi=-\boldsymbol{M}_{d} \boldsymbol{H}+\lambda \boldsymbol{M}_{d} \boldsymbol{M}_{f}-\boldsymbol{M}_{f} \boldsymbol{H}-K_{f} \frac{\left(\boldsymbol{M}_{f} \boldsymbol{n}\right)^{2}}{M_{f}^{2}}-K_{d} \frac{\left(\boldsymbol{M}_{d} \boldsymbol{n}\right)^{2}}{M_{d}^{2}},
$$

where $\lambda$ is the intersublattice exchange constant, $\boldsymbol{n}$ is the direction of the easy axis and $K_{f, d}$ are the anisotropy constants for $f$ - and $d$-sublattices, respectively. The spin dynamics in ferrimagnet is described by Euler-Lagrange equations of the form $\frac{\mathrm{d}}{\mathrm{d} t} \frac{\partial \mathcal{L}}{\partial \dot{q}_{i}}-\frac{\partial \mathcal{L}}{\partial q_{i}}=-\frac{\partial \mathcal{R}}{\partial \dot{q}_{i}}$, where $q_{i}$ are the ordering angles.

When magnetic field $H$ is smaller than the intersublattice exchange field $H_{\text {ex }}$, which is of the order of $50 \mathrm{~T}$, the canting angles between the sublattices are small. We simplify the further description by taking this into account. For this, let us introduce the Neel vector $\boldsymbol{L}=\boldsymbol{M}_{f}-\boldsymbol{M}_{d}$ and the net magnetization $\boldsymbol{M}=\boldsymbol{M}_{f}+\boldsymbol{M}_{d}$ vectors. In the vicinity of the compensation point the difference between the sublattice magnetizations $\left|M_{f}-M_{d}\right| \ll L$ is small. The two vectors are parameterized using the sets of angles $\theta, \varepsilon$ and $\varphi, \beta$, which are defined as:

$$
\begin{aligned}
\theta_{f} & =\theta-\varepsilon, \theta_{d}=\pi-\theta-\varepsilon, \\
\varphi_{f} & =\varphi+\beta, \varphi_{d}=\pi+\varphi-\beta .
\end{aligned}
$$

The schematic of the angles can be found in figure S1 in the supplementary data (available online at stacks.iop.org/ JPhysCM/32/01LT01/mmedia). Similarly to [21] we use the quasi-antiferromagnetic approximation to describe the dynamics near the magnetization compensation point. In this approximation the canting angles are small $\varepsilon \ll 1, \beta \ll 1$, and we can expand the Lagrangian (1) as well as the corresponding thermodynamic potential up to quadratic terms in small variables. In this case the antiferromagnetic vector is naturally defined as $\boldsymbol{L}=(L \sin \theta \cos \varphi, L \sin \theta \sin \varphi, L \cos \theta)$ and the expansions yield:

$$
\begin{aligned}
\mathcal{L} & =-\frac{m}{\gamma} \dot{\varphi} \cos \theta-\frac{\mathfrak{M}}{\gamma} \sin \theta(\dot{\varphi} \varepsilon-\beta \dot{\theta})-\Phi, \\
\Phi & =-K(\boldsymbol{l}, \boldsymbol{n})^{2}-H m \cos \theta-\varepsilon \mathfrak{M} H \sin \theta+\frac{\delta}{2}\left(\varepsilon^{2}+\sin ^{2} \theta \beta^{2}\right) .
\end{aligned}
$$

Here $m=M_{f}-M_{d}, \mathfrak{M}=M_{f}+M_{d}, K=K_{f}+K_{d}$ is the effective uniaxial anisotropy constant, $\boldsymbol{l}=\boldsymbol{L} / L$ is the unit Neel vector $\delta=2 \lambda M_{d} M_{f}$. In the final expression for the thermodynamic potential we assume that the anisotropy 
is weak $K \ll \lambda \mathfrak{M}$. For $\mathrm{GdFeCo}$ with $24 \% \mathrm{Gd}$ and compensation point near $283 \mathrm{~K}$, we take the following values: $\mathfrak{M} \approx 1700 \mathrm{emu} \mathrm{cm}^{-3}, K=1.5 \times 10^{5} \mathrm{erg} \mathrm{cm}^{-3}, \lambda=18.5 \mathrm{~T} / \mu_{B}$, $\delta \approx 2.5 \times 10^{9} \mathrm{erg} \mathrm{cm}^{-3}$. The characteristic values of small angles $\epsilon$ and $\beta$ are of the order of $10^{-2}$.

Next, we exclude the variables $\varepsilon$ and $\beta$ by solving the Euler-Lagrange equations $\frac{\mathrm{d}}{\mathrm{d} t} \frac{\partial \mathcal{L}}{\partial \dot{\varepsilon}}-\frac{\partial \mathcal{L}}{\partial \varepsilon}=-\frac{\partial \mathcal{R}}{\partial \dot{\varepsilon}} \approx 0$ and $\frac{\mathrm{d}}{\mathrm{d} t} \frac{\partial \mathcal{L}}{\partial \dot{\beta}}-\frac{\partial \mathcal{L}}{\partial \beta}=-\frac{\partial \mathcal{R}}{\partial \dot{\beta}} \approx 0$. The solutions are:

$$
\begin{aligned}
& \varepsilon=\frac{\mathfrak{M}}{\delta} \sin \theta\left(H-\frac{\dot{\varphi}}{\gamma}\right), \\
& \beta \sin \theta=\frac{\mathfrak{M}}{\delta} \frac{\dot{\theta}}{\gamma} .
\end{aligned}
$$

Substituting them into the Lagrangian (5), we obtain the effective Lagrangian, which describes the dynamics of a uniaxial ferrimagnet in the vicinity of the compensation point:

$$
\begin{aligned}
\mathcal{L}_{\text {eff }}= & \frac{\chi_{\perp}}{2}\left(\frac{\dot{\theta}}{\gamma}\right)^{2}+m \cos \theta\left(H-\frac{\dot{\varphi}}{\gamma}\right) \\
& +\frac{\chi_{\perp}}{2} \sin ^{2} \theta\left(H-\frac{\dot{\varphi}}{\gamma}\right)^{2}+K(\boldsymbol{l}, \boldsymbol{n})^{2}, \\
\Phi_{\text {eff }}(H)= & -m H \cos \theta-\frac{\chi_{\perp}}{2} H^{2} \sin ^{2} \theta-K(\boldsymbol{l}, \boldsymbol{n})^{2}, \\
& \mathcal{R}_{\text {eff }}=\frac{\alpha \mathfrak{M}}{2 \gamma}\left(\dot{\theta}^{2}+\sin ^{2} \theta \dot{\varphi}^{2}\right)
\end{aligned}
$$

where $\chi=\frac{2 \mathfrak{M}^{2}}{\delta}$. In GdFeCo $\chi \approx 3 \times 10^{-3}$ and $\alpha \approx 0.05$. In the derivation above we assumed the gyrotropic factor $\gamma$ and Gilbert damping constant $\alpha$ to be the equal for both sublattices. Taking into account the difference between the values of $\gamma_{i}$ and $\alpha_{i}(i=f, d)$ for the two sublattices will lead to the angular momentum compensation effect at certain temperature. The Lagrangian, Rayleigh function and equations of motion preserve the same form in this case if we substitute the parameters $\gamma$ and $\alpha$ with temperature-dependent factors $\widetilde{\gamma}$ and $\widetilde{\alpha}$ defined as:

$$
\begin{aligned}
& \frac{1}{\widetilde{\gamma}}=\frac{1}{\bar{\gamma}}\left(1-\frac{\mathfrak{M}}{m} \frac{\gamma_{f}-\gamma_{d}}{\gamma_{f}+\gamma_{d}}\right)=\frac{\frac{M_{d}}{\gamma_{d}}-\frac{M_{f}}{\gamma_{f}}}{\left(M_{d}-M_{f}\right)}, \\
& \frac{1}{\bar{\gamma}}=\frac{1}{2}\left(\frac{1}{\gamma_{d}}+\frac{1}{\gamma_{f}}\right), \widetilde{\alpha}=\frac{\left(\alpha_{d} \gamma_{f}+\alpha_{f} \gamma_{d}\right)}{\left(\gamma_{f}+\gamma_{d}\right)} \frac{1}{1+\frac{\mathfrak{M}}{m} \frac{\gamma_{f}-\gamma_{d}}{\gamma_{f}+\gamma_{d}}} .
\end{aligned}
$$

The expression is able to reproduce the features of laser induced spin dynamics of ferrimagnet in a collinear phase near the angular momentum compensation as reported in [15] and studied theoretically in [7].

\section{Excitation of the spin dynamics}

The proposed approach appears to be a powerful tool allowing to explore the magnetization dynamics in ferrimagnets that occurs in the noncollinear phase. Let us consider the following example that still seeks for theoretical explanation. A femtosecond laser pulse excites a uniaxial ferrimagnet (for instance, it can be $\mathrm{GdFeCo}$ or $\mathrm{TbFeCo}$ ) in the presence of external static magnetic field. The impact of the laser pulse leads to the demagnetization of one or both of the sublattices. What is the sub-100 ps dynamics in the magnetic system that follows the demagnetization?

In our framework the spin dynamics in ferrimagnet is described by Euler-Lagrange equations with regard to the polar and azimuthal angles $\theta$ and $\varphi$ that describe the orientation of the antiferromagnetic vector $\boldsymbol{L}$. Let us consider a particular case when the easy magnetization axis is aligned with the external magnetic field, which leads to the presence of azimuthal symmetry in the system. In this case $\boldsymbol{n}=(0,0,1)$. In this case the Euler-Lagrange equations can be rewritten as:

$$
\frac{\chi_{\perp}}{\gamma^{2}} \ddot{\theta}=\frac{\partial \mathcal{L}_{\mathrm{eff}}}{\partial \theta}-\frac{\partial \mathcal{R}_{\mathrm{eff}}}{\partial \dot{\theta}}, \frac{\mathrm{d}}{\mathrm{d} t} \frac{\partial \mathcal{L}_{\mathrm{eff}}}{\partial \dot{\varphi}}=-\frac{\partial \mathcal{R}_{\mathrm{eff}}}{\partial \dot{\varphi}} .
$$

The nonlinear equations that are similar to equation (11) and describe the spin dynamics of two-sublattice ferrimagnets were obtained in the work [22] under the conditions $H=0$ and $\mathcal{R}_{\text {eff }}=0$.

From Lagrangian (8) we see that the moment of inertia of the system along the easy axis (EA) $I_{\mathrm{EA}}=\frac{\chi \perp}{2} \sin ^{2} \theta$ is zero in the collinear phase. Therefore, no precession is expected as a result of demagnetization if initially the system resides in the collinear phase. Next, we consider the response in the noncollinear phase. Over the short time of demagnetization the second equation can be approximately treated as a conservation law and the conserving quantity (angular momentum of magnetization precession $\mathcal{J}$ ) stays approximately constant as $\partial \mathcal{L} / \partial \varphi=0$ due to the Noether theorem:

$\mathcal{J}=\frac{\partial \mathcal{L}_{\text {eff }}}{\partial \dot{\varphi}}=-\frac{1}{\gamma}\left[m \cos \theta+\chi_{\perp} \sin ^{2} \theta\left(H-\frac{\dot{\varphi}}{\gamma}\right)\right]=$ const.

Let the moment of time $t=0-$ denote the moment just before the excitation with the laser pulse and assume that before the excitation the ferrimagnet is in the ground state defined as $\theta(0-)=\theta_{0}, \varphi(0-)=\varphi_{0}$, and $\dot{\varphi}(0-)=0, \dot{\theta}(0-)=0$. At different values of the the temperature and magnetic field the ferrimagnet might reside in one of the two possible antiferromagnetic collinear phases or in noncollinear phase. The phase transitions between the states may be represented on the phase diagram in H-T coordinates [13]. If the ultrafast laser-induced demagnetization is small and the changes of $M_{f}, M_{d}$ and $\mathfrak{M}$ are of the order of percent or less, the change of $m$ (which is approximately equal to the total magnetization near the compensation point) may be still dramatic and it can even change its sign near the compensation temperature. In what follows, we assume that the demagnetization is associated only with a change of $m$, namely $m=m_{0}+\Delta m(t)$. As we will see below, the change in this quantity leads to drastic effects in dynamics.

Therefore, the conservation law (12) leads to the emergence of azimuthal dynamics $\dot{\varphi}(t)$ at the demagnetization timescales $(\Delta t)$ due to demagnetization $\Delta m(t)$ :

$$
\dot{\varphi}(t)=\gamma \frac{\Delta m(t)}{\chi_{\perp}} \frac{\cos \theta_{0}}{\sin ^{2} \theta_{0}} .
$$


This expression describes the precessional response in the noncollinear phase, where $0<\theta_{0}<\pi$. Note, that, equivalently, this phase is characterized by the canting of the sublattices. There exists a correspondence between total tilt resulting in $\theta \neq 0, \pi$ and canting, which follows from equation (6). Emergence of the azimuthal spin precession as a result of demagnetization of the medium is similar to the well-known Einstein-de-Haas effect, where the demagnetization leads to azimuthal precession of the magnet. Subsequently, this azimuthal spin dynamics leads to the emergence of polar dynamics $\theta(t)$, which is most commonly measured in pump-probe experiments of ultrafast magnetism, by acting as an effective field $H_{\text {eff }}=H-\frac{\dot{\varphi}}{\gamma}$ in the Lagrangian (5). We can then view the Lagrangian as depending only on variable $\theta$ and the effective field $H_{\text {eff }}$. At demagnetization $\delta m \sim 0.01 \mathrm{M}$ in $\mathrm{GdFeCo}$ the value of $\dot{\varphi}$ can reach up to $1 \mathrm{THz}$, and the corresponding effective magnetic field is of the order of $10 \mathrm{~T}$. Note that initial state of the system corresponds to the condition $\frac{\partial \Phi}{\partial \theta_{0}}\left(H_{\text {eff }}=H\right)=0$. We can rewrite the Euler-Lagrange equation from equation (11) for polar angle as follows:

$$
\frac{\chi_{\perp}}{\gamma^{2}} \ddot{\theta}+\frac{\partial \Phi\left(H_{\mathrm{eff}}\right)}{\partial \theta}=-\frac{\alpha \mathfrak{M}}{\gamma} \dot{\theta}
$$

Or, alternatively:

$\frac{\chi_{\perp}}{\gamma^{2}} \ddot{\theta}+m \sin \theta H_{\mathrm{eff}}-\chi_{\perp} \sin \theta \cos \theta\left(H_{\mathrm{eff}}^{2}-\frac{2 K}{\chi_{\perp}}\right)=-\frac{\alpha \mathfrak{M}}{\gamma} \dot{\theta}$.

By integrating this equation over the demagnetization time $\Delta t$ we obtain the state of system after the laser pulse at $t=0+$, which is characterized by the initial conditions

$$
\theta(0+)=\theta_{0}, \dot{\varphi}(0+), \varphi(0+)=\int_{0}^{\Delta t} \dot{\varphi}(t) \mathrm{d} t, \dot{\theta}(0+)=\int_{0}^{\Delta t} \ddot{\theta}(t) \mathrm{d} t .
$$

The value $\Delta t$ is of the order of the optical pulse length. After the excitation $(t=0+)$ the magnetization precession will start. Critical dynamics occurs near the second-order phase transitions to the collinear phases where $\theta=0, \pi$ as is seen from equation (13).

\section{Critical dynamics}

In a simple model case of ultrafast demagnetization and ultrafast recovery of magnetization with $\Delta m(t)=\Delta m$, $0<t<\Delta t ; \Delta m(t)=0, t>\Delta t$, we obtain the initial condition from (15):

$$
\begin{aligned}
\dot{\theta}(+0) \approx & {\left[-\left(2 \frac{\cos ^{2} \theta_{0}}{\sin \theta_{0}}+\sin \theta_{0}\right) H+\frac{m_{0}}{\chi_{\perp}} \frac{\cos \theta_{0}}{\sin \theta_{0}}+\frac{\Delta m}{\chi_{\perp}} \frac{\cos \theta_{0}}{\sin ^{3} \theta_{0}}\right] \frac{\gamma^{2}}{\chi_{\perp}} \Delta m \Delta t } \\
& =B\left(\theta_{0}\right) \Delta m+O\left(\Delta m^{2}\right) .
\end{aligned}
$$

This quantity defines the initial angular momentum of the polar spin precession that is induced in the system by the femtosecond

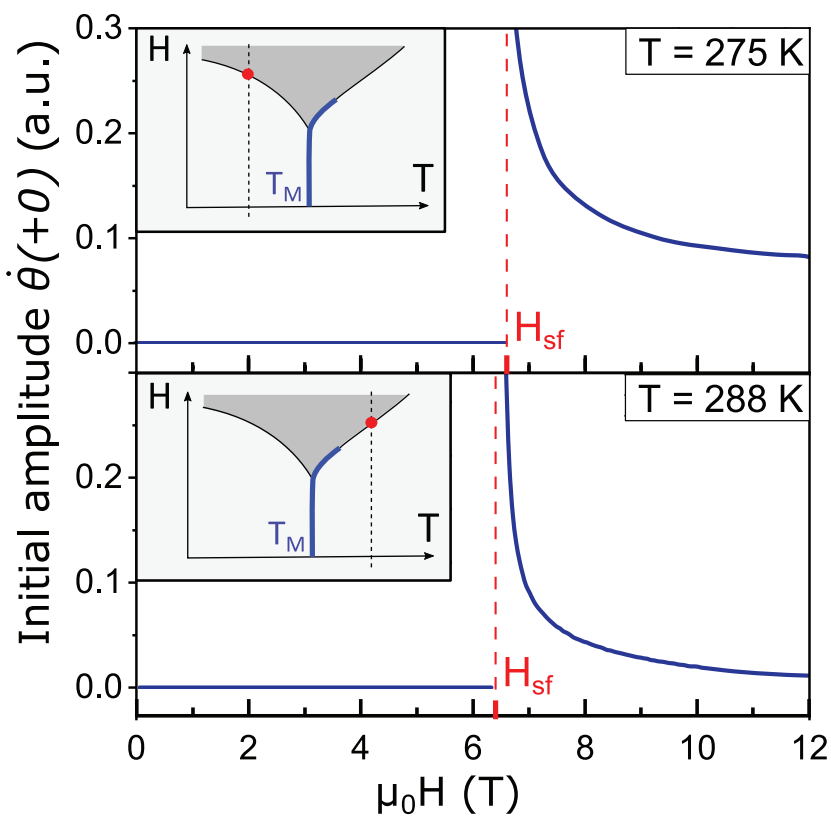

Figure 1. The amplitude of the magnetization precessional response after the demagnetization due to the femtosecond laser pulse action in $\mathrm{GdFeCo}$ ferrimagnet near the compensation point at different temperatures. Insertions: the schematic of the magnetic phase diagram. There are two antiferromagnetic collinear phases with $\boldsymbol{M}_{d}$ directed along (opposite) to the external magnetic field above (below) the compensation temperature $T_{M}$. Above them, an area where the noncollinear phase exists, which is filled with gray color. The blue line represents the first-order phase transition that occurs between two collinear phases in weaker magnetic fields and then, with the increase of the field, between one of the collinear phases and noncollinear one. The black solid lines are the secondorder phase transition lines. The dashed lines corresponds to the fixed temperature in the plot. The red dot is the point of phase transition for this temperature.

laser pulse. The amplitude of oscillations is proportional to the initial condition (16). Its dependence on the external magnetic field is illustrated in figure 1 for different temperatures for magnetic parameters of $\mathrm{GdFeCo}$ uniaxial ferrimagnet. At low values of external magnetic fields there is only collinear ground state in the ferrimagnet and above certain field $H_{\mathrm{sf}}$ the transition to a noncollinear state occurs [23]. The schematic of the magnetic phase diagram for $\mathrm{GdFeCo}$ is shown in inserts to figure 1 . At $T=275 \mathrm{~K}$ and $T=288 \mathrm{~K}$ the phase transitions are of the second order, corresponding to a smooth transition from angle $\theta_{0}=0$ to $\theta_{0}>0$. It is seen that the amplitude of the coherent magnetization dynamics launched by the ultrafast demagnetization diverges at $H_{\text {sf }}$. Note that just above the compensation temperature the transition is of the first order and the the magnetization dynamics is more complex. The behavior of the transition line is sensitive to the magnetic anisotropy of the material and has been studied by us in [24]. In the region of the firstorder phase transition critical divergence will be no longer seen. The features of the theoretically described critical slowdown were observed experimentally in [17]. The disappearance of the critical divergence right above the compensation point was not 
observed experimentally. Most likely, observation of this peculiarity requires a finer tuning of parameters in the experiment.

Another feature in the dynamics described by our model is the critical behavior of the characteristic timescales in the vicinity of the second-order phase transitions. To demonstrate this effect analytically, we first solve the equation for $\delta \theta(t)=\theta(t)-\theta_{0}$.

$$
\dot{\delta} \ddot{\theta}+\omega_{r}^{2}\left(\theta_{0}\right) \delta \theta=-\alpha \omega_{\mathrm{ex}} \delta \dot{\theta},
$$

where $\omega_{r}^{2}\left(\theta_{0}\right)=\gamma^{2}\left[\frac{m}{\chi_{\perp}} H \cos \theta_{0}+\left(\frac{2 K}{\chi_{\perp}}-H^{2}\right) \cos 2 \theta_{0}\right], \omega_{\mathrm{ex}}=\gamma \frac{\mathfrak{M}}{\chi_{\perp}}$. The initial condition is $\theta(0)=\theta_{0}$; another one is given by equation (16). In the limit of low amplitude oscillations and taking into account that in the vicinity of the second-order phase transition $\omega_{r}<\alpha \omega_{\mathrm{ex}} / 2$ the solution has the form $\delta \theta(t)=A e^{-\nu t} \sinh \omega t$, where $\nu=\alpha \omega_{\mathrm{ex}} / 2, \omega^{2}=\nu^{2}-\omega_{r}^{2}$, $A=B\left(\theta_{0}\right) / \omega$. The rise time can be estimated from the condition $\dot{\theta}\left(\tau_{\text {rise }}\right)=0$ :

$$
\tau_{\text {rise }} \approx \frac{\operatorname{atanh} \frac{\omega}{\nu}}{\omega}=\frac{\operatorname{atanh} \frac{\sqrt{\nu^{2}-\omega_{r}^{2}}}{\nu}}{\sqrt{\nu^{2}-\omega_{r}^{2}}} .
$$

The characteristic time of the oscillations decay (relaxation time) is proportional to the imaginary part of eigenfrequency and can be estimated by the following expression:

$$
\tau_{\text {relax }} \approx \frac{4 \pi \nu}{\omega_{r}^{2}} .
$$

Near the second-order phase transition the mode softening occurs and the eigenfrequency turns to zero: $\omega_{r} \rightarrow 0$, and we observe growth of the both timescales. The critical behavior of the rise time was observed in GdFeCo experimentally [17] and the typical values of $\tau_{\text {rise }}$ were of the order of $10 \mathrm{ps.}$

\section{Conclusions}

To sum up, the developed theoretical model based on the quasi-antiferromagnetic Lagrangian formalism proved to be suitable for description of the sub-100 ps laser-induced magnetization dynamics in rare-earth - transition metal (RE-TM) ferrimagnets near the compensation point. We have found that laser-induced demagnetization can result in a torque acting on sublattice magnetizations that is non-zero in a noncollinear phase only. We have explained the experimentally observed critical behavior of the response amplitude and the characteristic timescales. The anomalous behavior is a consequence of the magnetic field-induced second-order phase transition from collinear to a noncollinear phase. These effects are especially pronounced in the vicinity of the compensation point. Understanding the ultrafast response to demagnetizing optical or electrical pulses and subsequent spin dynamics can facilitate the future developments in the fields of ultrafast magnetism, magnonics and spintronics.

\section{Acknowledgments}

This research has been supported by RSF Grant No. 17-1201333. MDD and AVK acknowledge support from the Netherlands Organization for Scientific Research (NWO).

\section{ORCID iDs}

M D Davydova (은ttps://orcid.org/0000-0001-8482-5832

\section{References}

[1] Kirilyuk A, Kimel A V and Rasing T 2010 Rev. Mod. Phys. 822731

[2] Beaurepaire E, Merle J C, Daunois A and Bigot J Y 1996 Phys. Rev. Lett. 764250

[3] Stanciu C D, Hansteen F, Kimel A V, Kirilyuk A, Tsukamoto A, Itoh A and Rasing T 2007 Phys. Rev. Lett. 99047601

[4] Moreno R, Ostler T A, Chantrell R W and ChubykaloFesenko O 2017 Phys. Rev. B 96014409

[5] Ostler T et al 2012 Nat. Commun. 3666

[6] Wienholdt S, Hinzke D, Carva K, Oppeneer P M and Nowak U 2013 Phys. Rev. B 88020406

[7] Schlickeiser F, Atxitia U, Wienholdt S, Hinzke D, Chubykalo-Fesenko O and Nowak U 2012 Phys. Rev. B 86214416

[8] Chimata R et al 2015 Phys. Rev. B 92094411

[9] Atxitia U, Ostler T, Barker J, Evans R F L, Chantrell R W and Chubykalo-Fesenko O 2013 Phys. Rev. B 87224417

[10] Khorsand A R, Savoini M, Kirilyuk A, Kimel A V, Tsukamoto A, Itoh A and Rasing T 2012 Phys. Rev. Lett. 108127205

[11] Radu I et al 2011 Nature 472205

[12] Clark A E and Callen E 1968 J. Appl. Phys. 395972

[13] Zvezdin A 1995 Handbook of Magnetic Materials (Elsevier:New Holland) pp 405-543 (https://www. elsevier.com/books/handbook-of-magnetic-materials/ buschow/978-0-444-82232-1)

[14] Stanciu C D, Tsukamoto A, Kimel A V, Hansteen F, Kirilyuk A, Itoh A and Rasing T 2007 Phys. Rev. Lett. 99217204

[15] Stanciu C D, Kimel A V, Hansteen F, Tsukamoto A, Itoh A, Kirilyuk A and Rasing T 2006 Phys. Rev. B 73220402

[16] Chen Z, Gao R, Wang Z, Xu C, Chen D and Lai T 2010 J. Appl. Phys. 108023902

[17] Becker J, Tsukamoto A, Kirilyuk A, Maan J C, Rasing T, Christianen P C M and Kimel A V 2017 Phys. Rev. Lett. 118117203

[18] Wilson R B, Gorchon J, Yang Y, Lambert C H, Salahuddin S and Bokor J 2017 Phys. Rev. B 95180409

[19] Yang Y, Wilson R B, Gorchon J, Lambert C H, Salahuddin S and Bokor J 2017 Sci. Adv. 3

[20] Liebs M, Hummler K and Fähnle M 1992 Phys. Rev. B 4611201

[21] Zvezdin A 1979 JETP Lett. 28553 (http://www.jetpletters. ac.ru/ps/1456/article_22180.shtml)

[22] Ivanov B and Sukstanskii A 1983 Zh. Eksp. Teor. Fiz. 84370

[23] Goransky B and Zvezdin A 1969 JETP Lett. 10196

[24] Davydova M, Zvezdin K, Becker J, Kimel A and Zvezdin A 2019 Phys. Rev. B 100064409 